\title{
High-definition vertically aligned liquid crystal microdisplays using a circularly polarized light
}

\author{
Kuan-Hsu Fan-Chiang \\ Shu-Hsia Chen \\ Shin-Tson Wu \\ University of Central Florida
}

Find similar works at: https://stars.library.ucf.edu/facultybib2000 University of Central Florida Libraries http://library.ucf.edu

This Article is brought to you for free and open access by the Faculty Bibliography at STARS. It has been accepted for inclusion in Faculty Bibliography 2000s by an authorized administrator of STARS. For more information, please contactSTARS@ucf.edu.

\section{Recommended Citation}

Fan-Chiang, Kuan-Hsu; Chen, Shu-Hsia; and Wu, Shin-Tson, "High-definition vertically aligned liquid crystal microdisplays using a circularly polarized light" (2005). Faculty Bibliography 2000s. 5178.

https://stars.library.ucf.edu/facultybib2000/5178

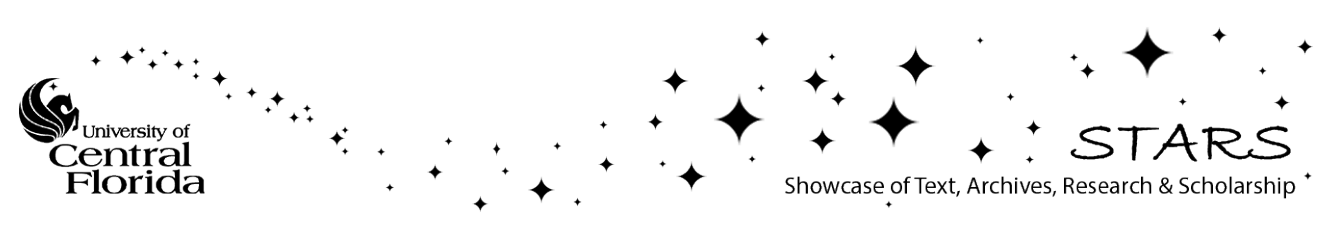




\title{
High-definition vertically aligned liquid crystal microdisplays using a circularly polarized light
}

\author{
Kuan-Hsu Fan-Chiang ${ }^{\text {a) }}$ and Shu-Hsia Chen \\ Department of Photonics and Institute of Electro-Optical Engineering, National Chiao-Tung University, \\ Hsinchu 300, Taiwan, Republic of China \\ Shin-Tson Wu \\ College of Optics and Photonics, University of Central Florida, Orlando, Florida 32816
}

(Received 8 February 2005; accepted 20 June 2005; published online 14 July 2005)

\begin{abstract}
A high-definition vertically aligned liquid crystal (LC) microdisplay exhibits a excellent contrast ratio, but its fringing field effect splits the bright state unevenly and leads to a very slow response time. By utilizing a circularly polarized light instead of conventional linearly polarized light, we have overcome the long-standing problems of poor sharpness, low brightness, and slow response time. Confirming computer simulations agree with the experimental results well. This approach can be applied to both reflective and transmissive LC microdisplays. (C) 2005 American Institute of Physics. [DOI: 10.1063/1.1999837]
\end{abstract}

Liquid crystal (LC) microdisplays have been widely used for data projectors, large-screen rear-projection televisions (RPTVs), and near-to-eye (NTE) virtual projections. ${ }^{1}$ Several active matrix technologies ${ }^{2}$ have been developed, including high temperature polycrystalline silicon (HTPS), low temperature polycrystalline silicon (LTPS), and liquidcrystal-on-silicon (LCOS) devices. The ever-increasing demands on high resolution and high contrast ratio lead to small pixel sizes $(\sim 10 \mu \mathrm{m})$ and interpixel gaps. As the interpixel gap is reduced, the lateral components of the fringing fields generated by the voltage difference between the adjacent pixels play a critical role to the optical performance. The influences of the fringing-field-effect in different LC modes have been investigated previously. ${ }^{3-5}$ Among them, the vertically aligned (VA) LCOS is particularly promising because of its inherent high contrast ratio. However, the light loss of a VA-LCOS due to the fringing-field-effect is relatively high $(\sim 30 \%)$ when a linearly polarized light in employed, ${ }^{3,6}$ this device is abbreviated as LPVA cell for convenience. Hence, the sharpness and brightness of the displayed images are deteriorated tremendously. Moreover, the optical transition time of the LPVA LCOS switched from the alternate bright and dark states to the all-bright state is extremely slow which results in blurred images.

In this letter, we demonstrate the circularly polarized light illuminated vertically aligned (CPVA) liquid crystal cell which not only preserves the light in the presence of the lateral fringing field but also gives a fast optical switching time $(<10 \mathrm{~ms})$ to eliminate the blurring of the moving images. We first simulate the LC directors distribution and optical performances of the CPVA and the LPVA cells and then apply the de Vries theory ${ }^{8}$ to interpret these optical properties. Finally, we present the confirming experimental results using a LCOS device.

Although our analyses are concentrated on the reflective LCOS devices because of their severe fringing-field-effect, these treatments can be applied equally well to the transmissive microdisplays, such as HTPS and LTPS panels. Figures 1(a) and 1(b) depict the optical configurations used for the CPVA and LPCA device studies, respectively. As shown in

${ }^{a)}$ Electronic mail: simonf.eo89g@nctu.edu.tw
Fig. 1(a), the incident light is linearly polarized by a polarizing beam splitter (PBS) along the $\mathrm{x}$ axis. The light will then traverse through the LC layer, whose rubbing direction deviates from the $\mathrm{x}$-axis by $45^{\circ}$. The reflective pixel electrodes fabricated in the device will reflect the light back to the LC layer. After passing the LC layer the second time, the outgoing light will be analyzed by the PBS and directed to the detector. For the CPVA device, a broadband circular polarizer is used instead of a PBS, as shown in Fig. 1(b). Therefore, the incident light is circularly polarized and modulated by the LC cell. After traversing through the LC layer twice, the light will be analyzed by the same circular polarizer and directed to the detector. For the following simulations and experimental results, the LCOS panel is operated at the alternate bright and dark states with the on-state voltage $V_{\text {on }}$ $=5 \mathrm{~V}$ and the off-state voltage $V_{\text {off }}=0 \mathrm{~V}$. Here the turned-on voltage corresponds to the maximum reflectance. The pixel size is $11.5 \mu \mathrm{m}$, the interpixel gap is $0.5 \mu \mathrm{m}$, and the cell gap $d=2.6 \mu \mathrm{m}$. The LC pretilt angle $\alpha_{0}=88^{\circ}$ and the LC material used for simulations is Merck MLC-6608; its extraordinary refractive index $n_{e}=1.5578$, ordinary refractive index $n_{o}=1.4748$, and dielectric anisotropy $\Delta \varepsilon=-4.2$.

In order to study the relation between the director profile and the optical properties, the two-dimensional (2D) LC director distribution was calculated first by the commercial software 2dimMOS (from autronic-MELCHERS GmbH) using the finite-element method. The $2 \mathrm{D}$ reflectance profiles were then calculated by the Jones Matrix method. ${ }^{9,10}$ Figure 2 presents the simulated results of the LC director distribu-
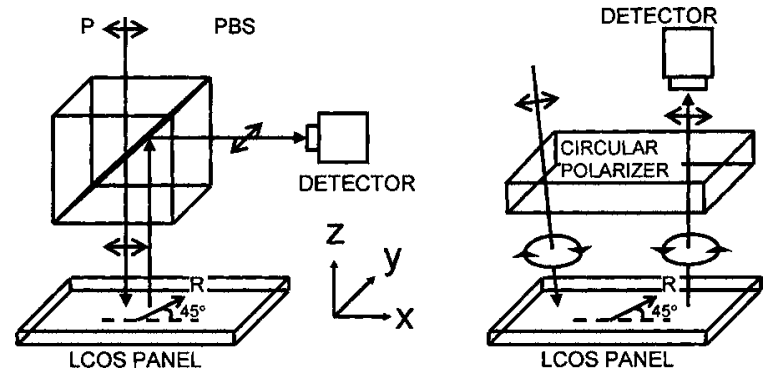

FIG. 1. Schematic drawing of the systems used for (a) the LPVA device and (b) the CPVA device. PBS=polarizing beam splitter; $\mathrm{P}=$ polarization axis, $\mathrm{R}$ is the $\mathrm{LC}$ alignment direction at the front surface. 


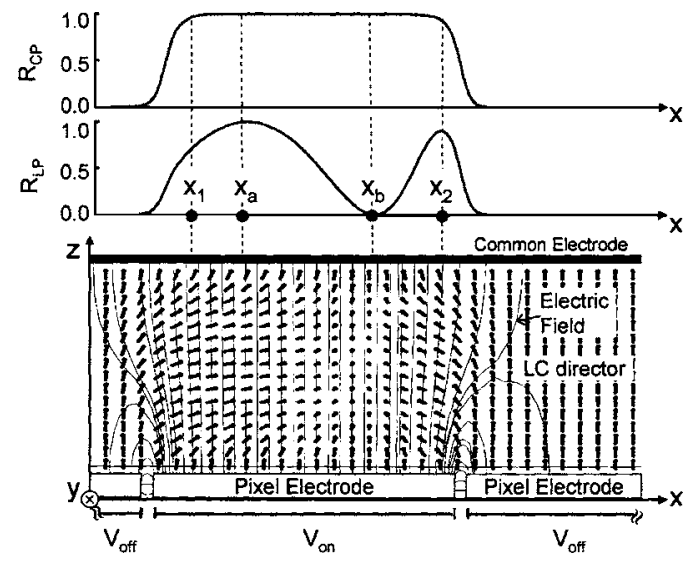

FIG. 2. Simulated LC director distribution and the corresponding reflectance profiles, $R_{\mathrm{LP}}(x)$ and $R_{\mathrm{CP}}(x)$, at the alternate dark and bright state for the CPVA and the LPVA systems, respectively. The on-state voltage $V_{\text {on }}=5 \mathrm{~V}$ and the off-state voltage $\mathrm{V}_{\text {off }}=0$.

tion and the corresponding reflectance profiles, $R_{\mathrm{CP}}(x)$ (top) and $R_{\mathrm{LP}}(x)$ (middle), at the dark-bright-dark state (bottom) for the CPVA and the LPVA systems and Fig. 3 shows the calculated azimuthal angle, $\phi$, of the LC directors along the $\mathrm{z}$ direction at the specified $\mathrm{x}$ positions, $\mathrm{x}=\mathrm{x}_{1}, \mathrm{x}_{\mathrm{a}}, \mathrm{x}_{\mathrm{b}}$, and $\mathrm{x}_{2}$ as denoted in Fig. 2. From Fig. 2, the fringing fields penetrate into the voltage-off pixels from both right and left edges of the voltage-on pixel. Without the electric field, the director $\mathbf{n}$ of this vertically aligned cell is parallel in a plane with an azimuthal angle of $45^{\circ}$ from the $\mathrm{x}$ axis. With the field on, the LC directors on the substrate surfaces are pinned due to the strong anchoring boundary condition. The largest polar angle appears near the middle plane because the negative type $(\Delta \varepsilon<0)$ LC molecules tend to orient themselves perpendicular to the electric field. Therefore, the director profile can be regarded as a screw-like structure with its helical axis along $\mathrm{z}$ axis and have opposite rotational sense in the upper and lower parts.

With regard to the optical properties, it is shown in Fig. 2 that there are two LC domains separated at $x_{b}$, where the dark line occurs on the bright pixel of the LPVA system. At $x_{b}$, nearly all the directors are aligned in the y direction. On the left and right sides, the directors have their maximum twist angles smaller and larger than $45^{\circ}$, respectively. In the LPVA cell, a reflectance peak is displayed in each domain, i.e., $R_{\mathrm{LP}}\left(x_{a}\right)$ and $R_{\mathrm{LP}}\left(x_{2}\right)$, while $R_{\mathrm{LP}}\left(x_{b}\right)$ displaying a minimum reflectance. The $R_{\mathrm{LP}}(x)$ is lying between $R_{\mathrm{LP}}\left(x_{a}\right)$ [which is $\left.\sim R_{\mathrm{LP}}\left(x_{2}\right)\right]$, and $R_{\mathrm{LP}}\left(x_{b}\right)$ in the rest area of the voltage-on pixel. The accumulated light loss is above $30 \%$. On the contrary, $R_{\mathrm{CP}}(x)$ is not influenced by the director distortions in the $\mathrm{x}-\mathrm{y}$ plane leading to a high sharpness and high brightness image in the CPVA cell.

As mentioned above, the LC director profile can be regarded as a screw-like structure with spatially varying pitch, $\mathrm{P}(\mathrm{z})$. Some studies have been made in such structures with different models of $\mathrm{P}(\mathrm{z}){ }^{11,12}$ Here we interpret the optical properties qualitatively by de Vries theory ${ }^{8}$ for simplicity. Assuming a wave propagates along the helical axis of an ideal helical structure with a fixed pitch $P_{0}$. In general, there are two eigenwaves propagating in the same direction. Their eigenvalues $l_{i}, i=1,2$, satisfy the dispersion relation which can be derived from solving the Maxwell's equations supplemented by the constitutive equation: ${ }^{13,14}\left(-k_{0}^{2}+l_{i}^{2}+q_{0}^{2}\right)^{2}$ $-4 q_{0}^{2} l_{k}^{2}-k_{1}^{4}=0$, where $k_{0}^{2}=(2 \pi / \lambda)^{2}\left(n_{e}^{2}+n_{o}^{2}\right) / 2, k_{1}^{2}=(2 \pi / \lambda)^{2}$

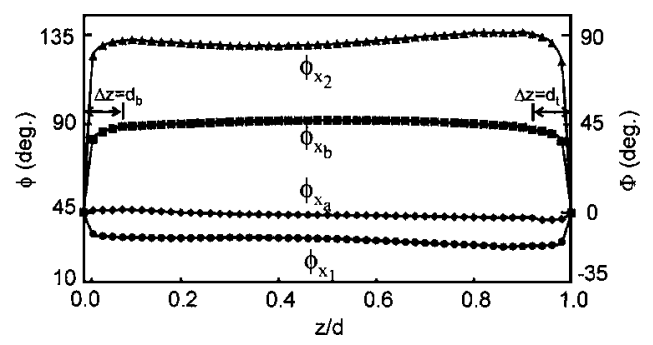

FIG. 3. Calculated azimuthal angle, $\phi$, and twist angle, $\Phi=\phi-45^{\circ}$, of the LC directors along the $\mathrm{z}$ direction at $x=x_{1}, x_{a}, x_{b}$, and $x_{2}$ as denoted in Fig. 2.

$\left(n_{e}^{2}-n_{o}^{2}\right) / 2, q_{0}=2 \pi / P_{0}$, and $\lambda$ is the wavelength of the incident light. When $P_{0}$ and $\lambda$ are known, one can obtain the ellipticity of the eigenwaves as:

$$
\rho=\frac{2 l_{i} q_{0}}{ \pm \sqrt{k_{1}^{4}+4 P_{0}^{2} l_{i}^{2}-k_{1}^{2}}},
$$

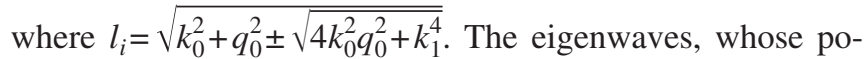
larization ellipse axes are either parallel or perpendicular to $\mathbf{n}$, have their phase difference accumulated to $\left(l_{1}-l_{2}\right) z$. From Eq. (1), if $P_{0} \gg \lambda \gg P_{0}\left(n_{e}-n_{o}\right)$, then $\rho \rightarrow \pm 1$. In this regime, the eigenwaves are almost circularly polarized, which means the oscillation direction of a linear wave will be rotated after passing the LC layer. The optical rotatory power (the amount of rotation per unit length) is given as $\psi=k_{1}^{4} /\left[8 q_{0}\left(k_{0}^{2}-q_{0}^{2}\right)\right]$. Also from Eq. (1), if $\lambda \ll P_{0}\left(n_{e}-n_{o}\right)$, then $\rho \rightarrow 0$ or $\infty$. In this regime, the eigenwaves are almost linearly polarized at the direction parallel or perpendicular to $\mathbf{n}$.

In the CPVA system, the light is preserved in the region of $x_{1}<x<x_{2}$ as presented by the reflectance profile $R_{c p}(x)$ in Fig. 2. This can be understood easily by the above arguments with an approximated model, which is a nontwisted LC layer sandwiched between two twisted layers. In Fig. 3, the twisted regimes near the substrates $\left(0 \leqslant z \leqslant d_{b}\right.$ and $d$ $\left.-d_{t} \leqslant z \leqslant d\right)$ have their effective pitch $\left|P_{0}\right|=\left[2 \pi / \Phi\left(d_{b}\right)\right] d_{b}$ $\cong\left[2 \pi / \Phi\left(d-d_{t}\right)\right] d_{t}$, where $\Phi(z)$ denotes the twist angle of the LC director at $z$ and is defined as the difference of azimuthal angles between that LC director and the surface LC director. By using Eq. (1), the eigenwaves in these regimes are almost circularly polarized for the visible light. The incident circularly polarized light remains its polarization state after passing the top-twisted regime. Note that a circularly polarized light can be regarded as the superposition of any two orthogonal linearly polarized waves with the same amplitude and $\pi / 2$ phase difference. The nontwisted uniform regime $\left(d_{b}<z<d-d_{t}\right)$, as shown in Fig. 3, with linearly polarized eigenwaves provides a phase difference, say $\delta=\pi / 2$, such that the resulted light is linearly polarized in the direction at $45^{\circ}$ from $\mathbf{n}$. The reflected light that traverses through the bottom-twisted regime twice (forward and backward) will have the same linear polarization. Then the uniform regime changes it to be circularly polarized by gathering another $\pi / 2$ phase change. The polarization state of the outgoing circular wave will again not be influenced after traversing through the top-twisted regime. Finally, almost all of the light passes through the broadband circular polarizer. In other words, the CPVA system preserves the input light efficiently.

On the contrary, for the LPVA system the input light is linearly polarized in the $\mathrm{x}$ direction. The top-twisted regime 


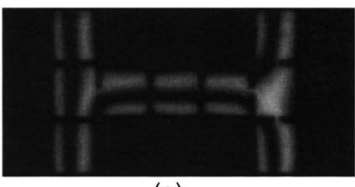

(a)

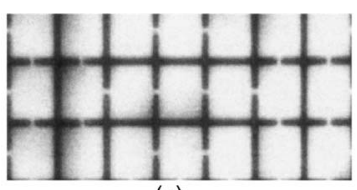

(c)

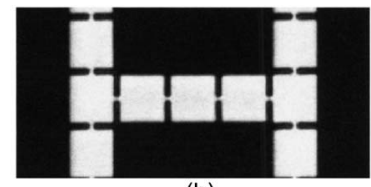

(b)

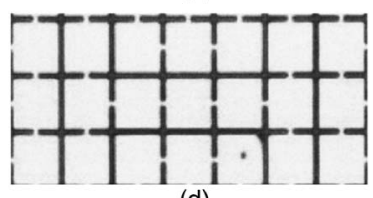

(d)
FIG. 4. Images captured by CCD through the polarizing microscope of the LC panel operated at the alternate bright and dark state for (a) the LPVA and (b) the CPVA devices. Images of the LC panel switched from the alternate bright and dark state to the all-bright state for (c) the LPVA device at $198 \mathrm{~ms}$ after switching and (d) the CPVA device at $33 \mathrm{~ms}$ after switching.

will slightly rotate the plane of polarization. The nontwisted regime will generally change the light to an elliptically polarized light. The characteristic of the polarization ellipse depends on the angle between the local director $\mathbf{n}$ and the entering linear wave. At $x=x_{b}$, most directors are aligned parallel in the $y$ direction. Since the incident light of the nontwisted region is almost linearly polarized in the $\mathrm{x}$ direction, one of the linear eignewaves has almost zero amplitude while the other remains oscillating in the $\mathrm{x}$ direction after having passed through this region. The reflected light that traverses through the bottom twisted regime twice will maintain the same polarization. i.e., along the $\mathrm{x}$ direction. The light will then traverse through the uniform and the toptwisted regions the second time with its polarization almost unchanged as already discussed above. Eventually, the outgoing light will be blocked by the analyzer resulting in a minimum reflectance, $R_{\mathrm{LP}}\left(x_{b}\right)$. At $x=x_{a}$ as shown in Fig. 3, most directors without twist deformation are aligned in the $45^{\circ}$ direction with respect to the $\mathrm{x}$ axis. Therefore, the two linear eigenwaves have almost the same amplitudes in this regime. By gathering a $\pi$ phase change after passing through the LC layer twice, the outgoing light will be linearly polarized at the direction nearly perpendicular to that of the incident light, which results in a maximum reflectance $R_{\mathrm{LP}}\left(x_{a}\right)$. The reflectance for the rest area can be deduced from the same model and the results are in between $R_{\mathrm{LP}}\left(x_{a}\right)$ and $R_{\mathrm{LP}}\left(x_{b}\right)$.

Figures 4(a) and 4(b) depict the experimental results when the panel is operated at the alternating bright and dark states for the LPVA and the CPVA devices, respectively. The images were captured by CCD through an optical polarizing microscope. It is clearly shown that the image of the LPVA device is degraded by the fringing-field-effect severely. The dark lines in the bright pixels originate from the distortion of the LC director profile illustrated in Figs. 2 and 3. By contrast, Fig. 4(b) shows that the dark lines are totally eliminated in the CPVA device. Therefore, the sharpness and the brightness of the displayed images are enhanced significantly.

The CPVA optical system not only improves the static performance but also dramatically reduces the dynamic response time of the LCOS panel. Figures 4(c) and 4(d) show the photos of the LC panel switched from the alternate bright and dark states to the all-bright state for the LPVA and the CPVA devices, respectively. The elapsed time after switching

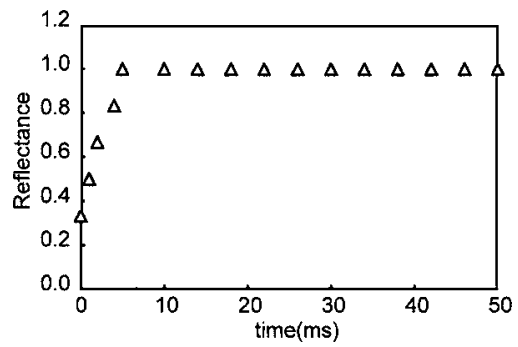

FIG. 5. Measured reflectance with respect to the elapsed time after switching from the alternate bright and dark state to the all-bright state for the CPVA device.

is $198 \mathrm{~ms}$ in Fig. 4(c) and $33 \mathrm{~ms}$ in Fig. 4(d). The distorted LC directors take an extremely long time to relax back to the original state. The dark lines in the LPVA device last for at least several hundred milliseconds after switching which causes a serious image blurring effect. Since the distorted LC directors are mainly rotating in the $x-y$ plane during the relaxation process, the influence on the optical response of the CPVA device is very small. Figure 5 shows the measured reflectance with respect to the elapsed time after switching from the alternate bright and dark states to the all-bright state for the CPVA device. The result indicates that the transition time is less than $10 \mathrm{~ms}$.

In conclusion, we have demonstrated a circularly polarized light illuminated vertically aligned LCOS microdisplay. The outstanding problems of poor sharpness and low brightness in the conventional device illuminated by a linearly polarized light are overcome. Furthermore, the dynamic transition time of the CPVA device switched from the alternate bright and dark states to the all-bright state is less than 10 $\mathrm{ms}$, which is adequate to eliminate the blurring of moving images in the LPVA device. The CPVA system preserves the same high contrast ratio $(>1000: 1)$ as the LPVA. The optical properties are interpreted qualitatively by the de Vries theory. Potential applications of this device for high contrast and high optical efficiency reflective LCOS and transmissive p-Si TFT-LCD projection displays are foreseeable.

This work was partially supported by the National Science Council, R.O.C., under Contract No. NSC 93-2112-M009-022. The authors are indebted to Dr. Xinyu Zhu (UCF) for useful discussions and Himax Technologies, Inc., for offering the experimental samples.

${ }^{1}$ E. H. Stupp and M. S. Brennesholtz, Projection Displays (Wiley, New York, 1998).

${ }^{2}$ E. Lueder, Liquid Crystal Displays (Wiley, New York, 2001).

${ }^{3}$ K. H. Yang and M. Lu, Displays 20, 211 (1999).

${ }^{4}$ H. De Smet, J. Van den Steen and A. Van Calster, SID Tech. Dig. 32, 968 (2001).

${ }^{5}$ K. H. Fan Chiang, S. T. Wu, and S. H. Chen, Jpn. J. Appl. Phys., Part 1 41, 4577 (2002).

${ }^{6}$ Y. Ji, J. Grandhi and M. E. Stefanov, SID Tech. Dig, 30, 750 (1999).

${ }^{7}$ S. Zhang, M. Lu, and K. H. Yang, SID Tech. Dig, 31, 898 (2000).

${ }^{8}$ H. de Vries, Acta Crystallogr. 4, 219 (1951).

${ }^{9}$ R. C. Jones, J. Opt. Soc. Am. 31, 488 (1941).

${ }^{10}$ S. T. Wu and D. K. Yang, Reflective Liquid Crystal Displays (Wiley, New York, 2001).

${ }^{11}$ Z. Lu, L. Li, H. Vithana, Y. Jiang and S. M. Faris, Mol. Cryst. Liq. Cryst. Sci. Technol., Sect. A 301, 237 (1997).

${ }^{12}$ S. Kutter and M. Warner, Eur. Phys. J. E 12, 515 (2003).

${ }^{13}$ P. G. de Gennes and J. Prost, The Physics of Liquid Crystals (Clarendon, Oxford, 1993).

${ }^{14}$ P. C. Yeh and C. Gu, Optics of Liquid Crystal Displays (Wiley, New York, 1999). 